\title{
Expanded microchannel heat exchanger: Non-destructive evaluation
}

Heat Transfer Engineering, 2018

David C Denkenberger ${ }^{1, *}$, Michael J Brandemuehl ${ }^{2}$, Joshua M Pearce ${ }^{3}$ and John Zhai $^{2}$

${ }^{1}$ Department of Civil and Architectural Engineering, Tennessee State University, 3500 John A Merritt Boulevard, Nashville, TN 37209, USA.

Email: ddenkenb@tnstate.edu, Telephone no.615-963-5419, Fax no.615-963-5413

${ }^{2}$ Civil, Environmental, and Architectural Engineering Department, University of Colorado at Boulder, Boulder, CO, USA.

Email: Michael.Brandemuehl@ colorado.edu

Email: John.Zhai@colorado.edu

${ }^{3}$ Department of Materials Science \& Engineering and the Department of Electrical \& Computer Engineering, Michigan Technological University, Houghton, MI, USA.

Email: pearce@mtu.edu
Abstract
Recent theoretical developments in expanded microchannel polymer-based heat ex- changers were promising, but the initial experiments underperformed simple theory. In order to understand this discrepancy, this paper introduces a nondestructive methodol- ogy for characterizing polymer heat exchangers. A computerized tomography (X-ray) 
scan was performed to diagnose the problem. The method was tested on the expanded microchannel polymer heat exchanger to determine the variations in geometry between the theoretical and experimental heat exchanger. Channels were found to have variable heights causing flow maldistribution. The results are discussed to guide further technological development of this approach to heat exchanger design and fabrication and lays the groundwork for an advanced discretized modeling.

\section{Introduction}

Heat exchangers (HXs) are widespread. Though polymers have low strength and thermal conductivity, they can be used for low temperature, low pressure applications. Furthermore, the low cost and high corrosion resistance is advantageous in certain applications.

One advantageous way of designing HXs is to use microchannels. Microchannel HXs (typically $<1 \mathrm{~mm}$ hydraulic diameter) have decreased material cost, weight, and volume, but current fabrication techniques are expensive. Commercially available microchannel HXs use one of the following techniques: etching, LIGA (lithography, electroplating and molding), micromachining, and stereolithography [1]. Recent work [2] has introduced a potentially lowcost method of fabricating microchannel HX for polymer HXs.

Following on this development, this paper summarizes the expanded microchannel HX design, fabrication, and preliminary tests, which were significantly below the performance predicted by simple $N T U$ theory (see below). In order to understand this discrepancy, this paper introduces an original nondestructive methodology for characterizing polymer 
HXs. The method was tested on a polymer expanded microchannel HX (EMHX) to determine the variations in geometry between the theoretical and experimental HX. This is the main objective of the work. The results are discussed to guide further technological development of this approach to HX design and fabrication.

The HX variable introduction below is based on McQuiston, et al. [3]. The thermal goodness of a HX is described by its effectiveness, $\eta$, defined as the realized heat transfer rate as a fraction of the ideal heat transfer rate

$$
\boldsymbol{\eta}=\frac{\dot{q}}{\dot{q}_{\max }}
$$

where the realized heat transfer rate is

$$
\dot{q}=C\left(T_{h i}-T_{h o}\right)=C\left(T_{c o}-T_{c i}\right)
$$

where the temperature of the hot fluid going into the $\mathrm{HX}$ is $T_{h i}$, the temperature of the hot fluid coming out of the HX is $T_{h o}$, the temperature of the cold fluid going into the $\mathrm{HX}$ is $T_{c i}$, and the temperature of the cold fluid coming out of the HX is $T_{c o}$. The focus in this paper is on balanced flow, so the heat capacity rate is

$$
C=\dot{m} C_{p}
$$

where the mass flow rate $\dot{m}$ and the specific heat is $C_{p}$.

The maximum heat transfer rate is

$$
\dot{q}_{\max }=C\left(T_{h i}-T_{c i}\right)
$$


for a counter-flow HX with balanced flow,

$$
\eta=\frac{N T U}{N T U+1}
$$

where $N T U$ is "number of transfer units"

$$
N T U=\frac{h A}{C}
$$

where $A$ is the heat transfer area and $h$ is the heat transfer coefficient [3].

The idea of the EMHX is high-precision joining layers of material and later expansion [4]. The joining pattern (e.g. Figure 1) can be achieved by laser welding. The expansion could be achieved by inflation (stretching or non-stretching of the layers). The materials could be polymers, composites, ceramic precursors (polymers fired into ceramics), or metals. Mass production, e.g. with a roll-to-roll process, could enable low cost [5]. Low cost would facilitate high surface area and/or multiple stages to produce high effectiveness. Low pressure drop can be achieved with lower velocity and numerous parallel paths, especially for laminar flow.

\section{Methods}

\section{Fabrication}

An EMHX was fabricated by laser welding $28 \mu \mathrm{m}$ thick low density polyethylene (LDPE) sheets following the patterns shown in Figure 1. The pattern on the left allows the e.g. hot 
flow to enter on the bottom left of the pattern, and exit on the top right of the pattern. The pattern on the right allows the cold flow to enter on the top and exit at the bottom. These patterns alternate such that hot and cold fluids alternate. There were five hot layers and five cold layers, for a total of 35 channels in each direction. The expansion, using inflation, resulted in 2.1 mm hydraulic diameter channels. Clear polyvinyl chloride (PVC) tubes were coupled with the manifold to allow water flow. To maintain expansion, a rigid acrylic (poly methyl methacrylate: PMMA) frame was glued to the top and bottom of the EMHX.

The core and the manifolds were formed in the same expansion process (see Figure 1). The bent pattern has less welded area near the ends, so the "chessboard" pattern in the EMHX core is converted to fluid sheets of alternating hot and cold in the manifold. Note that the rectangular shape of the passages in Figure 2 would occur if the expansion were achieved by pulling the top and the bottom of the EMHX. Because expansion was achieved by the injection of air, the actual channels are more hexagonal, as shown with the 3-D X-ray scans in Figures 10 and 11. Next the cross-flow manifold separates the two flows by having either the end or side closed (welded). When the patterns are superimposed, no lines cross because this would create a weld all the way through the EMHX, preventing expansion there. The core of the heat exchanger was $10 \mathrm{~cm}$ long, $3 \mathrm{~cm}$ wide, and $1 \mathrm{~cm}$ high. The manifolds of the heat exchanger were $3 \mathrm{~cm}$ by $3.5 \mathrm{~cm}$ and variable height.

For the rest of this paper, effectiveness will be used in the temperature sense, rather than heat flux 


$$
\eta_{h}=\frac{T_{h i}-T_{h o}}{T_{h i}-T_{c i}}
$$

and

$$
\eta_{c}=\frac{T_{c o}-T_{c i}}{T_{h i}-T_{c i}}
$$

An average effectiveness was calculated from the experimental data. The $N T U$ was calculated for the prototype by assuming it was fully expanded with a Nussult number of 3.6. This yielded a heat transfer coefficient of $1000 \mathrm{~W} /(\mathrm{mK})$ with a hydraulic diameter of $2.1 \mathrm{~mm}$. The data are plotted according to this (see Figure 3). The vertical error bars are in the range of $0.4 \%$ to $1.4 \%$ and correspond to the uncertainty in temperature measurement. The error in the temperature measurement is composed of the error in the voltage sensor, switch, cold junction compensation, the thermocouple itself, logger resolution, and calibration. The overall error for an absolute temperature measurement assuming that all the errors are independent was calculated to be $\sim 0.5^{\circ} \mathrm{C}$. The percent error for each of the data points was calculated. On this plot, it would not be possible to distinguish the $\sim 1.5 \%$ uncertainty in NTU (produced by the $1.5 \%$ uncertainty in flow rate driven largely by changes in head). This shows that the variation in flow rate has a negligible influence on the uncertainty in effectiveness. The fall in effectiveness at intermediate flow rate is because the flow is significantly unbalanced, so averaging the effectivenesses does not produce the same result as balanced flow at the same average $N T U$. During the effectiveness experiments, the EMHX was horizontal. More details on 
the experiment can be found in [2]. The discrepancy between experimental data and model value increases as $N T U$ increases because the heat loss to the environment increases as a percent as the flow rate decreases (higher $N T U$ ). Since the experimental results were significantly below that predicted by the $N T U$ theory, it was necessary to determine the geometry.

\section{Methods for Measuring EMHX Geometry}

Flow maldistribution may have caused the significant reduction in effectiveness from the $N T U$ model. One possibility of determining the EMHX geometry would be to destructively test the EMHX. However, it is likely that the destruction process would distort the shape of the channels. Using an infrared camera would not work because the carbon black in the LDPE would make it a surface temperature measurement, so the internal details would not be revealed. The same problem would be faced by florescent methods. Magnetic particle inspection requires a ferrous material.

A number of nondestructive techniques have been used to image non-polymer heat exchangers, including thermal neutron radiography [6], ultrasound [7], Computerized Tomography (CT: 3-D X-ray) [8], magnetic resonance imaging (MRI) [9], and eddy current (not possible for polymers) [10]. However, little if any nondestructive evaluation has been performed on polymer HXs. It is possible that millimeter wave scanning or ultrafast optical pulses could work to determine the EMHX geometry. The ideal would be setting up flow with tracers and taking images at different times to get flow rate in each channel. However, 
the water flow takes days to set up, which would be very expensive in terms of equipment time.

A Medtronics “O arm” 3-D X-ray scanning machine shown in Figure 4 was used to determine the geometry of the EMHX. The X-ray contrast between water and LDPE is small, so the EMHX was drained. Also, dry air was pumped through the EMHX with a hand pump $(\sim 100 \mathrm{~mL} / \mathrm{s})$ for several hours, until the relative humidity dropped at the outlet. This indicated that the water in the EMHX was mostly evaporated, though there were some bumps in the Xray scan, possibly indicating some un-evaporated water in channels that received less air flow.

It was difficult to resolve the EMHX layers, so the EMHX was put in water to reduce the reflection of the X-rays off the top and bottom plates. After the $\mathrm{O}$ arm testing was complete, dry air was pumped through the EMHX again for several hours. Then a CT scan was performed at Avista Adventist Hospital (see Figure 5). First a CT scan was performed with no liquid in the EMHX. It was again difficult to resolve the channels. Therefore, an iodine solution was injected into the upper side tube with the other tubes closed with gum (the HX was inclined for the CT scans - see Figure 6 through 11). The EMHX was tapped to try to remove bubbles. The scan used $120 \mathrm{kV}$ and $78 \mathrm{~mA}$. In the "Extr soft tissue" and "Extr 3-D volume" modes, the picture was blurry (not shown). However, in the "Extr bone" mode, a relatively clear picture was generated. 
Next saline solution was added to the upper end tube to reveal a different flow path. The X-ray scattering cross-section of the saline solution was significantly lower than the iodine solution, so the two solutions could be differentiated (iodine white and saline grey).

\section{Results and Discussion}

Figure 6 throughFigure 11 are shown to characterize the geometry. The iodine solution outside the tube is explained by the fact that there is room between the LDPE and tube where fluid could infiltrate. All views are slightly off normal, so the entire length of a single channel is not visible. The lines below the EMHX are the towel.

Figure 6 shows CT scan in "Extr bone" mode side view with iodine solution put into upper side tube, which flowed into some channels. Moving across the EMHX exposed long bubbles in the iodine solution in the core (see Figure 7). Bubbles would likely be much less of a problem for actual EMHX operation, because the fluid could move through the EMHX, flushing bubbles out.

Moving further across the EMHX, Figure 8 shows the pinch in the center of the upper manifold, and also the channel pinches between the lower manifold and the core. The grey is the saline solution that was injected into the top end tube.

Next the CT scan in "Extr bone" mode cross sections was analyzed. Figure 9 shows part of the upper manifold, with the manifold pinch filled on top and bottom with adhesive (see also Figure 8) and alternating layers of saline solution. 
Three millimeters further down the EMHX, part iodine solution is in three straight channels and two bent channels (see Figure 10). This indicates an internal leak that could have been due to damage removing the heat exchanger from its container in the effectiveness measurement test set up. This figure also shows that the channel pattern is the same as in the core. This is unlike the prediction that the hot and cold flows would separate into discrete layers. However, the actual behavior is reasonable because the straight weld lines in the manifold (see Figure 1) would discourage contraction in the axial direction, and this contraction would have opened up the bent layers. Therefore, in future designs, only dots should be used in the manifold. The CT scan was also consistent with the observation that the EMHX did not contract in the axial direction upon expansion. Furthermore, the flow resistance was significantly higher for the bent direction, and this is consistent with having to overcome the pinches in flow presented by the manifold geometry.

In the core, a cross section shows saline and iodine to the left of the core, and adhesive to the right (see Figure 11). This demonstrates the predicted pattern similar to a chess board.

Despite the ability to see the channels better in most cases with the CT scan, it was not possible to reorient the images to be parallel to the channels. Therefore, the $\mathrm{O}$ arm scans were used for the measurements of the channel heights. A representative image is shown in Figure 12. A grid was drawn over the image to measure the height at 24 locations. Since there are seven channels per layer of LDPE, there are seven hot slices and seven cold slices. 
Since there is only one prototype, this could be significantly better or significantly worse effectiveness than the typical. However, at least in terms of the welds working, since there are 70 channels, there is fairly good statistical sampling.

If the channels are of different size, the fluid will flow quicker in some of the cold channels and thus the $N T U$ will be smaller, so the fluid will not warm up as much. An analogous effect also applies to the hot channels. These effects were explored by Ratts [11]. A further mechanism for producing channel irregularity is through non-uniform fouling, where solid material builds up randomly [12]. Still another way of producing channel variations is through two-phase flow, that is gases and liquids. This is usually found in condensers and evaporators. However, it also pertains to some water HXs because, as the water is heated, gasses come out of solution, forming bubbles. In addition, channel irregularity can be produced by thermally-induced flow maldistribution. This can occur if the temperature change in parallel layers is significantly different, which can arise due to different layers having different number of neighbors. Then if the fluid properties such as density and viscosity change much with temperature, the flow rate in different layers can be different [13]. Another channel irregularity category is the non-uniform spacing of fins [14].

An algebraic expression has been formulated for the ineffectiveness produced by flow maldistribution due to channel irregularities [15]. This is for the scenario of countercurrent shell in tube with no mixing, which may not exhibit the same behavior as the chessboard pattern this paper is investigating. Therefore, this correction is a good first approximation, but an 
advanced discretized model would be more accurate (to be published). The fractional diameter variation is described by $\pm a / 2$. An irregularity parameter for $C=1$, small diameter variations, high $N T U$, and laminar flow is

$$
\varepsilon=1-\frac{2 a}{3}
$$

Given $\varepsilon$, the effectiveness can be calculated for balanced flow:

$$
\boldsymbol{\eta}=\frac{\varepsilon\left(1-e^{(-N T U(1-\varepsilon))}\right)}{1-\varepsilon * e^{(-N T U(1-\varepsilon))}}
$$

The plot of equation 10 is shown in Figure 3 for several values of the irregularity parameter. As $N T U \rightarrow \infty, \eta \rightarrow \varepsilon$. However, at lower $N T U$, the loss in effectiveness due to flow maldistribution is smaller than the infinite $N T U$ case.

A number of works have discussed the flow maldistribution due to the manifold [1618]. Simply, not different paths of fluid in the manifold have different flow resistances, so the channels do not all have the same flow rates in the HX core. For very narrow manifolds, the manifold flow maldistribution issue can be extreme, even having reverse flow in some cases.

The best fit to experimental data was achieved with $\varepsilon=0.7$ (see Figure 3 ). This only applies to channel irregularity, and in reality, there was significant manifold flow maldistribution (and loss to the environment at high $N T U$ ). But still the fit with experimental data is dramatically improved with this channel flow maldistribution correction.

Overall, both X-ray machines were able to give approximately $0.6 \mathrm{~mm}$ resolution, allowing the determination of the geometry of the polymer EMHX with $2 \mathrm{~mm}$ channels. For 
significantly smaller channels, different equipment would be required. However, these machines would likely be adequate for the vast majority of polymer HXs from the channel size perspective. From a material perspective, since most other polymers are carbon-based, the Xray scattering would probably be greater because LDPE is one of the least dense polymers. Furthermore, for silicon-based polymers like silicone, the larger nucleus should provide even greater visibility. From a sheet thickness perspective, $28 \mu \mathrm{m}$ is relatively thin. This nondestructive evaluation of HXs could also be useful for assessing the health of polymer HXs in service, such as resilience to corrosion and fouling.

\section{Conclusions}

The expanded microchannel HX has the potential for low cost and high effectiveness. A particular application of the polymer version of this heat exchanger is in solar water pasteurization, which has the potential to save the lives of thousands of children a day $[19,20]$. However, for fabrication methods that result in imperfect expansion processes, an unknown geometry is formed. This paper provided a new nondestructive methodology utilizing X-ray imaging to determine the geometry. This provides a framework for other nondestructive tests of HXs. A finite difference model using this geometry has better predicted the effectiveness of the EMHX (to be published). 
Funding

American Society of Heating, Refrigeration, and Air Conditioning Engineers Graduate Research Fellowship and the University of Colorado at Boulder Technology Transfer Office Proof of Concept Grant provided financial support for this project.

Acknowledgements

Ray Radebaugh and Moncef Krarti provided valuable feedback. Jill Stone and Andrew Bour provided experimental assistance. The company Medtronics donated an $\mathrm{O}$ arm X-ray scan and Jerry Wall was the technician. Avista Adventist Hospital donated the CT scan and Chelle Flint and Jane were the technicians.

\section{Nomenclature}

a Fractional diameter variation is given by $\pm \mathrm{a} / 2$, dimensionless

A Total area for heat transfer, $\mathrm{m}^{2}$

C Heat capacity rate, $\mathrm{kJ} /(\mathrm{s} * \mathrm{~K})$

$\mathrm{C}_{\mathrm{p}} \quad$ Specific heat, $\mathrm{kJ} /(\mathrm{kg} * \mathrm{~K})$

EMHX Expanded microchannel HX

HX Heat exchanger, dimensionless

h Heat transfer coefficient, $\mathrm{W} /\left(\mathrm{m}^{2} * \mathrm{~K}\right)$

LDPE Low density polyethylene, dimensionless 
$\dot{\mathrm{m}} \quad$ Mass flow rate, $\mathrm{kg} / \mathrm{s}$

NTU Number of transfer units, dimensionless

PMMA Poly methyl methacrylate, dimensionless

PVC Polyvinyl chloride, dimensionless

$\dot{q} \quad$ Heat flux, $\mathrm{kW}$

$\mathrm{T} \quad$ Temperature, $\mathrm{K}$

\section{Greek Symbols}

$\varepsilon \quad$ Irregularity parameter, dimensionless

$\eta \quad$ Effectiveness, dimensionless

\section{Subscripts}

$\begin{array}{cl}\text { c } & \text { Cold fluid } \\ \text { h } & \text { Hot fluid } \\ \text { HT } & \text { Heat transfer } \\ \text { i } & \text { In } \\ \text { min } & \text { Minimum } \\ \text { max } & \text { Maximum } \\ \text { o } & \text { Out }\end{array}$

References

[1] S. Ashman, S. Kandlikar, “A Review of Manufacturing Processes for Microchannel Heat Exchanger Fabrication," presented at the 4th International Conference on Nanochannels, Microchannels and Minichannels, Limerick, Ireland, June 19-21, 2006. Informa UK Limited, issue 47608, pp. 855-860. DOI: 10.1115/ICNMM2006-96121.

[2] D. Denkenberger, M. Brandemuehl, J. Pearce, and J. Zhai, "Expanded microchannel heat exchanger: design, fabrication, and preliminary experimental test," Proc. Inst. Mech. 
Eng. Part A: J. Power Energy, vol. 226, no. 4, pp. 532-544, April 2012. DOI: $10.1177 / 0957650912442781$.

[3] F. McQuiston, J. Parker, and J. Spitler, Heating, ventilating, and air conditioning: analysis and design, $6^{\text {th }}$ ed. New York, NY, USA: Wiley 2005.

[4] D. Denkenberger, "Low-cost high-effectiveness microchannel heat exchanger: Fabrication, modeling, and validation," Ph. D. dissertation, Dept Civil, Environmental, and Architectural Engineering, University of Colorado at Boulder, Boulder, CO, USA, 2010.

[5] D. Denkenberger, "Microchannel expanded heat exchanger," U.S. Patent 9,618,278, Apr. $11,2017$.

[6] H. Asano, N. Takenaka, T. Fujii, H. Hayashi, and T. Wakabayashi, "Visualization and void fraction measurement of gas-liquid two-phase flow in a commercial plate heat exchanger by thermal neutron radiography," IEEE Trans On Nucl Sci, vol. 52, no. 1, pp. 280-284, Feb. 2005. DOI: 10.1109/TNS.2005.843676.

[7] M. Gori et al., "Guided waves by EMAT transducers for rapid defect location on heat exchanger and boiler tubes," Ultrasonics, vol. 34, no. 2-5 pp. 311-314, June 1996. DOI: 10.1016/0041-624X(95)00094-J.

[8] D. Kupperman, W. Deininger, N. Lapinski, C. Sciammarella, D. Yuhas, "Nondestructive Evaluation Techniques for Silicon Carbide Heat-Exchanger Tubing," presented at DARPA/AFML review of progress in quantitative NDE, La Jolla, CA, USA, 8-13, July, 1979. CONF-790757-3 TRN: 80-001250 DOE Contract Number: W-31-109ENG-38, chap 15, pp. 700-712, Jan. 1979.

[9] W. Wang, J. Walton, M. McCarthy, and K. McCarthy, "Evaluation of Mixing Profiles of Power Law Fluids in Scraped Surface Heat Exchanger Geometry Using MRI," in Spatially resolved magnetic resonance: methods, materials, medicine, biology, rheology, geology, ecology, hardware, P. Blumler, B. Blumich, R. Botto, and E. Fukushima, Eds. New York, NY, USA: Wiley, 1998 chap 52, pp. 539-545. DOI:

10.1002/9783527611843.ch52 
[10] G. Pichenot and T. Sollier, "Eddy Current Modelling for Nondestructive Testing," presented at the 8th European Conference on Non-Destructive Testing (ECNDT), Barcelona, Spain, June, 2002. Journal of Nondestructive Testing, vol. 8, no. 6, pp. 1-5, June 2003.

[11] E. Ratts, "Investigation of flow maldistribution in a concentric-tube, counterflow, laminar heat exchanger," Heat Transf. Eng., vol. 19, no. 3, pp. 65-75, 1998.

DOI: $10.1080 / 01457639808939927$.

[12] D. Bossan, J. Grillot, B. Thonon, and S. Grandgeorge, "Experimental study of particulate fouling in an industrial plate heat exchanger," J. Enhanc. Heat Transf., vol. 2, no. 1-2, pp. 167-175, 1995. DOI: 10.1615/JEnhHeatTransf.v2.i1-2.180.

[13] L. Haseler, "Layer Pattern Effects in Plate-Fin Heat Exchangers," Am. Soc. Mech. Eng. Heat Transf. Div., vol. 75, pp. 119-125, 1987.

[14] J. Mondt, "Effects of nonuniform passages on deepfold heat exchanger performance," J. Eng. Gas Turbines Power, vol. 99, no. 4, pp. 657-663, Oct. 1977. DOI:10.1115/1.3446564.

[15] M. Gotovsky et al., "Thermal effectiveness of heat exchangers: Effect of manufacturing inaccuracies and deformation of tube bundles," Heat Transf. Eng., vol. 6, no. 1, pp. 52-57, Jan. 1985. DOI: 1080/01457638508939619

[16] P. Heggs and H. Scheidat, "Thermal performance of plate heat exchangers with flow maldistribution," Am. Soc. Mech. Eng., New York, NY, USA, vol. 201, pp. 87-93, 1992.

[17] J. Wen and Y. Li, "Study of flow distribution and its improvement on the header of plate-fin heat exchanger," Cryogenics, vol. 44 no. 11, pp. 823-831, Nov. 2004. DOI: 10.1016/j.cryogenics.2004.04.009.

[18] F. Selimovic, T. Bruun, and B. Sundén, "Computational analysis of gas flow and heat transport phenomena in monolithic structures for high temperature processes," presented at the ASME 2005 Summer Heat Transfer Conference collocated with the ASME 2005 Pacific Rim Technical Conference and Exhibition on Integration and 
Packaging of MEMS, NEMS, and Electronic Systems, San Francisco, California, USA, July 17-22, 2005, Heat Transfer Eng., vol. 4, pp. 87-96. DOI:10.1115/HT200572183.

[19] S. Fukuda-Parr et al, Human development report 2001: Making new technologies work for human development. United Nations Development Programme, New York, NY, USA: Oxford University Press, 2001.

[20] J. Pearce and D. Denkenberger, "Numerical simulation of the direct application of compound parabolic concentrators to a single effect basin solar still," presented at the Proceedings of the Solar Cookers and Food Processing 2006 International Conference, Granada, Spain, Solar Cookers International, July 12-16, 2006.vol. 118, Jan. 2006. 


\section{List of Figure Captions}

Figure 1 Laser welding pattern (in white): left: "bent" channels where the, e.g., hot water enters the lower left side and exits the upper right side; right: "straight" channels where the, e.g., cold water enters the top and exits the bottom.

Figure 2 Cross section view of the expansion process for the HX core. The hot is flow into the page and cold is flow out of the page. The start is the top (black and green lines indicate material sheets and red lines indicate bonding) and the bottom is expanded (with insulation put on the outside).

Figure 3 NTU model, flow maldistribution (epsilon < 1) and experimental effectivenesses as a function of average NTU.

Figure 4 "O arm” 3-D X-ray machine at Medtronics in Louisville, CO, USA (EMHX at center).

Figure 5 CT scan machine at Avista Adventist Hospital in Louisville, CO, USA (EMHX at center on towels).

Figure 6 CT scan in "Extr bone" mode side view showing iodine solution (white) put into upper side tube and flowed into some channels. Also shown are the upper and lower plate, adhesive and channel walls.

Figure 7 CT scan in "Extr bone" mode side view showing bubbles in the iodine solution in the core.

Figure 8 CT scan in "Extr bone" mode with iodine and saline solutions showing fluid in the upper and lower end tubes, the pinch in the center of the upper manifold, and the channel pinches between the lower manifold and the core. 
Figure 9 CT scan in "Extr bone" mode with iodine and saline solutions showing a cross section of the upper manifold with the manifold pinch (see Figure 8) alternating layers of saline solution.

Figure 10 CT scan in "Extr bone" mode showing a cross section of the upper manifold demonstrating that the bent flow was pinched off.

Figure 11 CT scan in "Extr bone" mode showing a cross section of the core demonstrating the near chessboard pattern of iodine (white for one flow direction) and saline (grey for other flow direction). Some channels are empty (black), but nearly all 70 channels can be discerned.

Figure $12 \mathrm{O}$ arm scan parallel to channels with overlaid grid. Though the scan lines are very difficult to see, the core channel heights as a function of distance can be estimated. 

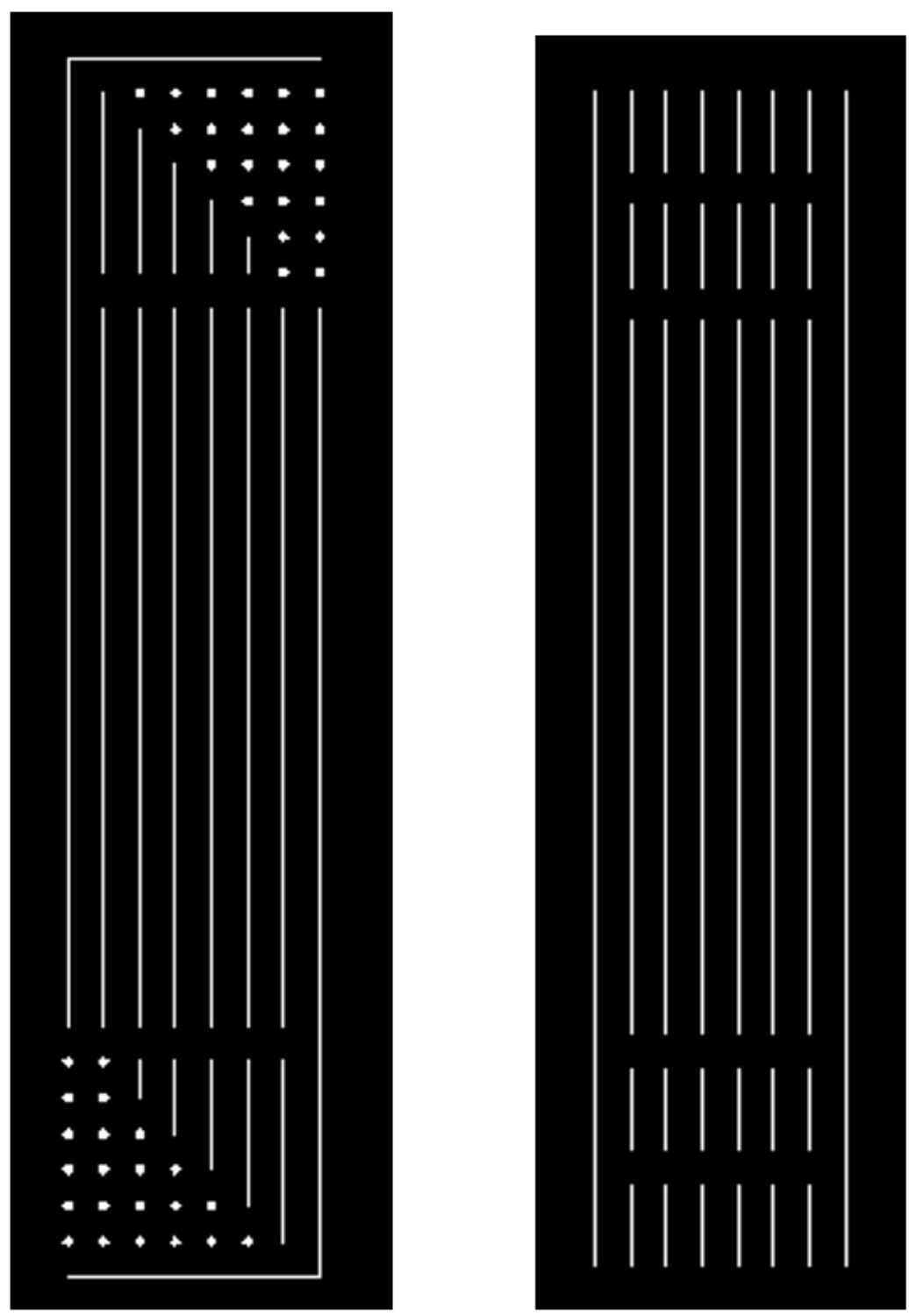

Figure 1 Laser welding pattern (in white): left: "bent" channels where the, e.g., hot water enters the lower left side and exits the upper right side; right: "straight" channels where the, e.g., cold water enters the top and exits the bottom. 

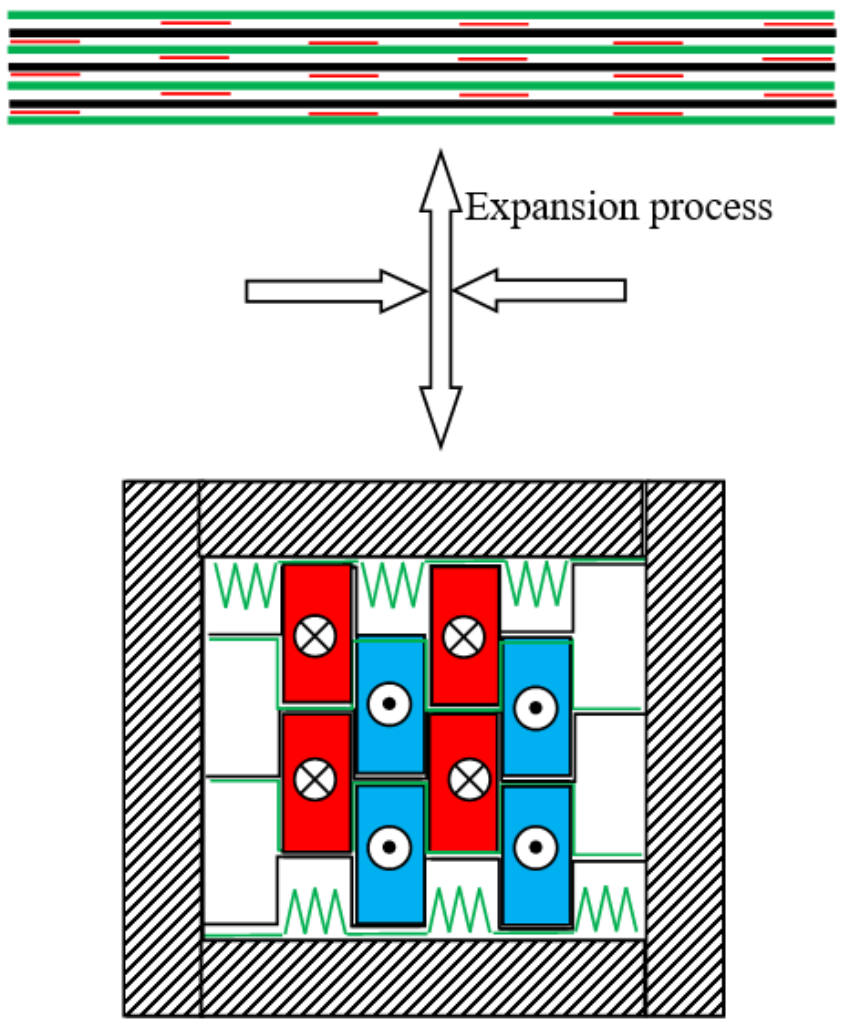

\begin{tabular}{l} 
Key \\
$\otimes$ Flow into page \\
$\odot$ Flow out of page \\
$\square$ Cold \\
$\square$ Hot \\
WA Insulation \\
W Loose Polymer \\
— Laser weld line \\
\hline
\end{tabular}

Figure 2 Cross section view of the expansion process for the HX core. The hot is flow into the page and cold is flow out of the page. The start is the top (black and green lines indicate material sheets and red lines indicate bonding) and the bottom is expanded (with insulation put on the outside). 


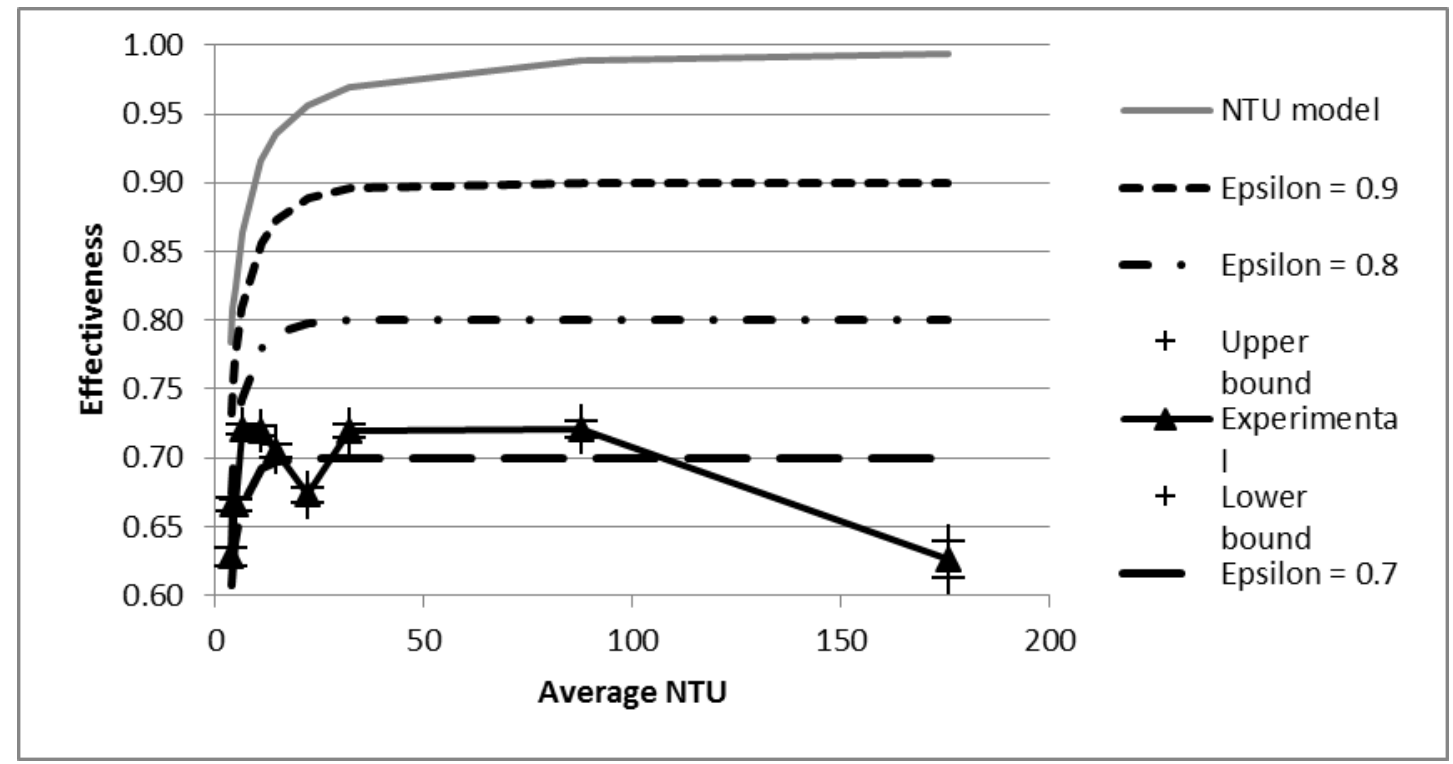

Figure 3 NTU model, flow maldistribution (epsilon < 1) and experimental effectivenesses as a function of average NTU. 


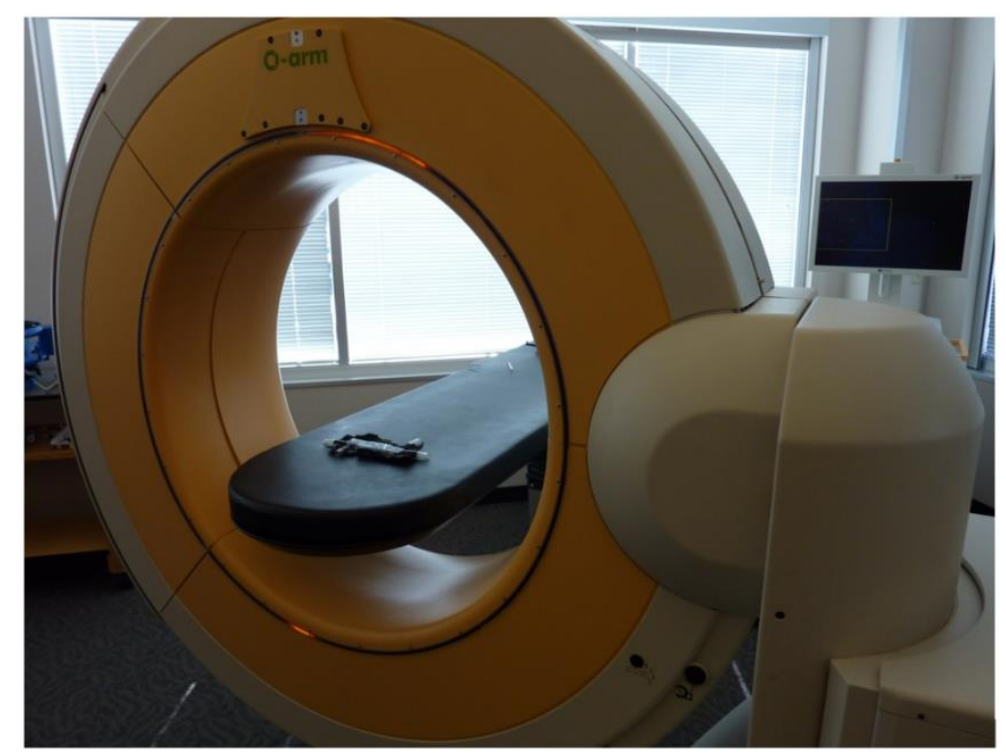

Figure 4 "O arm" 3-D X-ray machine at Medtronics in Louisville, CO, USA (EMHX at center). 




Figure 5 CT scan machine at Avista Adventist Hospital in Louisville, CO, USA (EMHX at center on towels). 


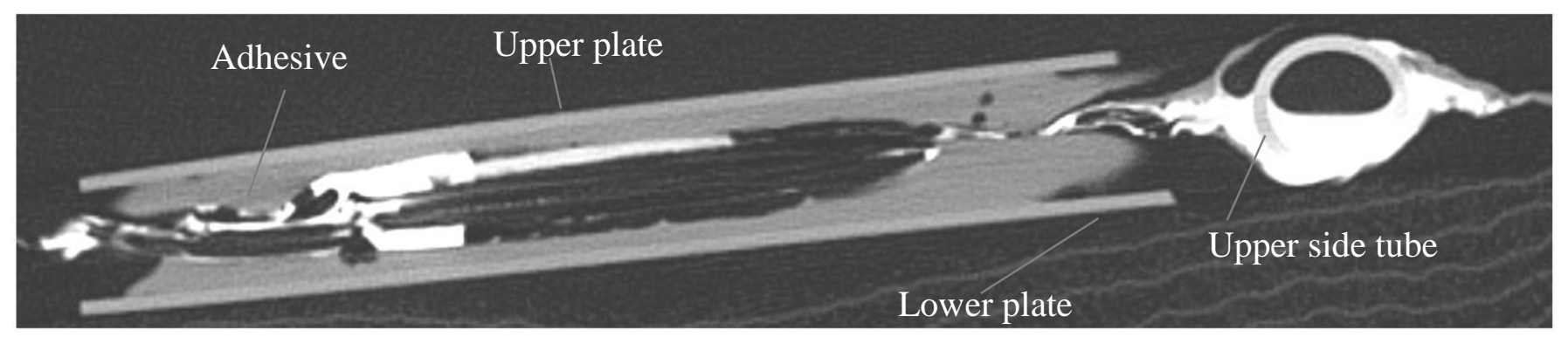

Figure 6 CT scan in "Extr bone" mode side view showing iodine solution (white) put into upper side tube and flowed into some channels. Also shown are the upper and lower plate, adhesive and channel walls. 


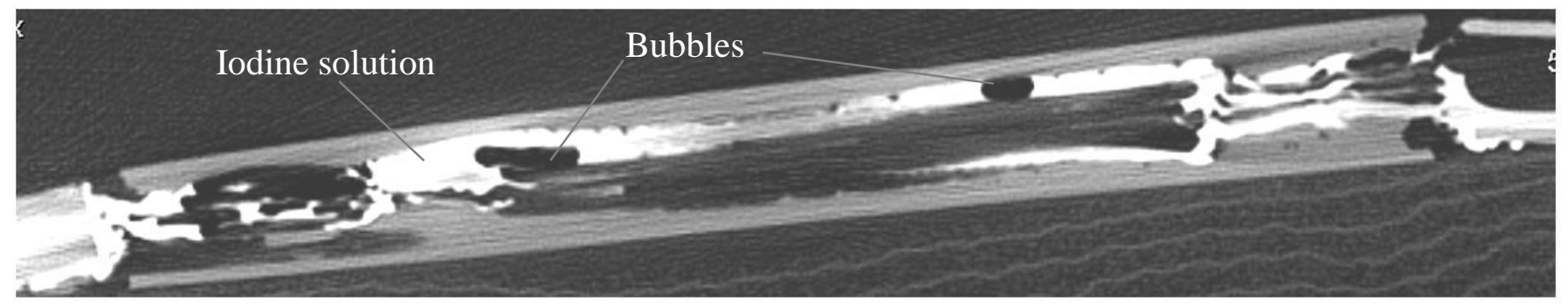

Figure 7 CT scan in "Extr bone" mode side view showing bubbles in the iodine solution in the core. 




Figure 8 CT scan in "Extr bone" mode with iodine and saline solutions showing fluid in the upper and lower end tubes, the pinch in the center of the upper manifold, and the channel pinches between the lower manifold and the core. 


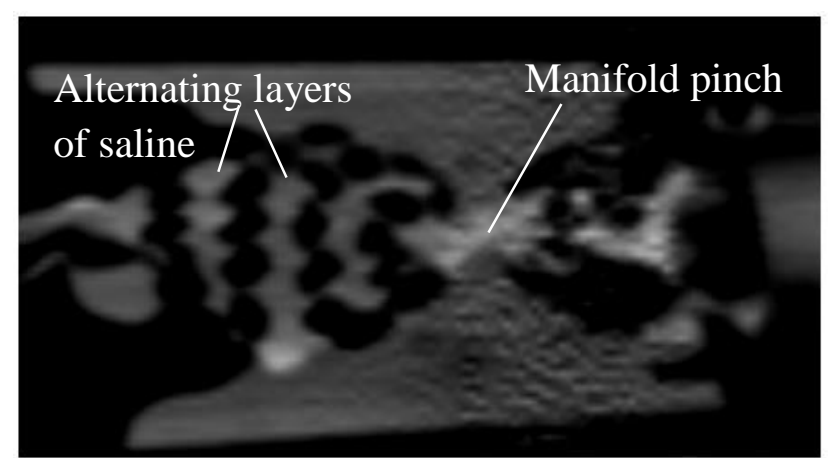

Figure 9 CT scan in "Extr bone" mode with iodine and saline solutions showing a cross section of the upper manifold with the manifold pinch (see Figure 8) alternating layers of saline solution. 


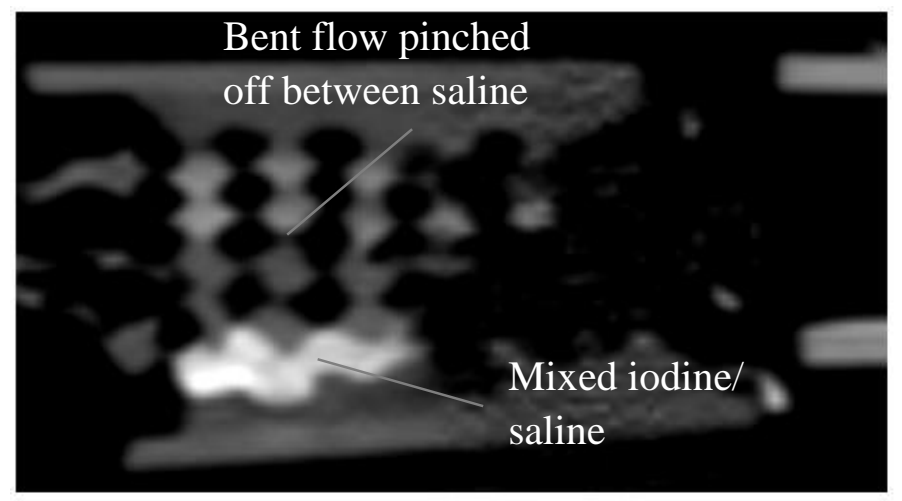

Figure $10 \mathrm{CT}$ scan in "Extr bone" mode showing a cross section of the upper manifold demonstrating that the bent flow that should have openings between the saline channels was pinched off. 




Figure 11 CT scan in "Extr bone" mode showing a cross section of the core demonstrating the near chessboard pattern of iodine (white for one flow direction) and saline (grey for other flow direction). Some channels are empty (black), but nearly all 70 channels can be discerned. 


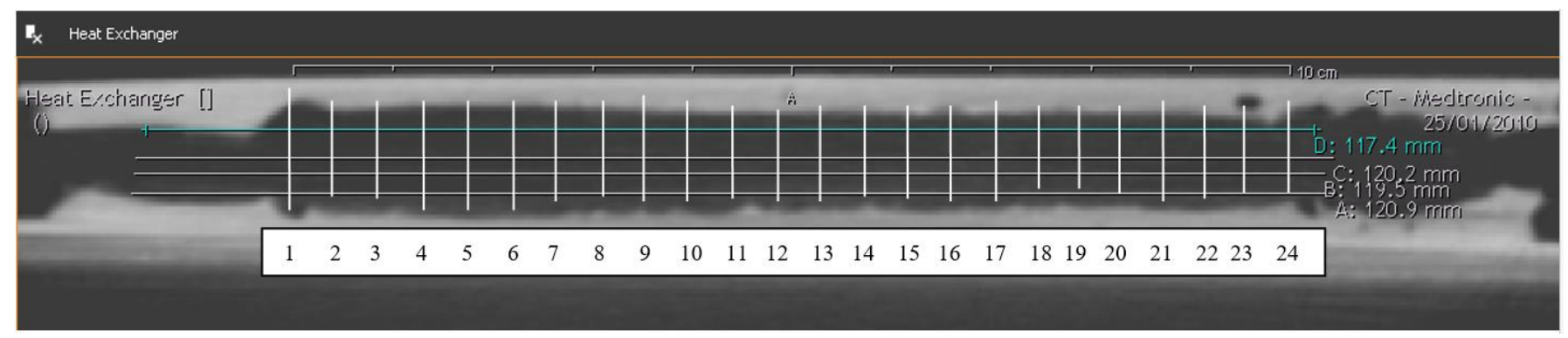

Figure $12 \mathrm{O}$ arm scan parallel to channels with overlaid grid. Though the scan lines are very difficult to see, the core channel heights as a function of distance can be estimated. 


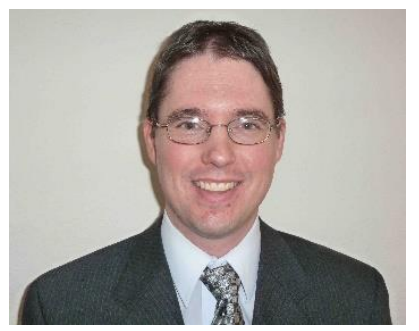

David Denkenberger received his B.S. from Penn State in Engineering Science, his M.S.E. from Princeton in Mechanical and Aerospace Engineering, and his Ph.D. from the University of Colorado at Boulder in the Building Systems Program. His dissertation was on his patented expanded microchannel heat exchanger. He is an assistant professor at Tennessee State University in architectural engineering. He is also an associate at the Global Catastrophic Risk Institute. He received the National Merit Scholarship, the Barry Goldwater Scholarship, the National Science Foundation Graduate Research Fellowship, is a Penn State distinguished alumnus, and is a registered professional engineer. He has authored or co-authored over 75 publications (over 1000 citations). He has given over 90 technical presentations.

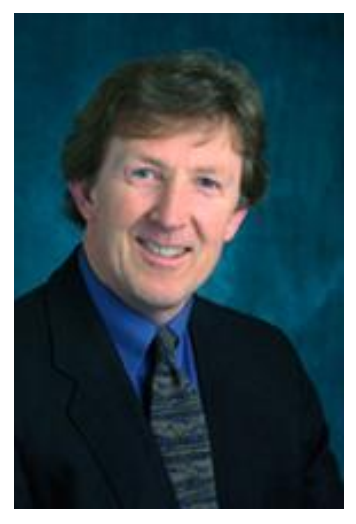

Michael J. Brandemuehl is Professor Emeritus in the Department of Civil, Environmental, and Architectural Engineering at the University of Colorado at Boulder. As a faculty in the Building Systems Program, he performed teaching and research related to the analysis, design, and operation of building energy systems. Specific interests and activities included integrated and sustainable building design, intelligent controls and building operations, building system simulation, and application of 
renewable energy technologies. He has been faculty advisor for the Solar Decathlon since its beginning. Prior to joining the faculty at Colorado, he was employed for seven years by United Technologies Carrier performing research and advanced product development for residential and commercial HVAC systems. He received his Ph.D. in Mechanical Engineering from the University of WisconsinMadison through the Solar Energy Laboratory; his dissertation involved heat and mass transfer in desiccant cooling systems.



Joshua M. Pearce received his Ph.D. in Materials Engineering from the Pennsylvania State University. He then developed the first Sustainability program in the Pennsylvania State System of Higher Education and helped develop the Applied Sustainability graduate engineering program while at Queen's University, Canada. He currently is a Professor cross-appointed in the Department of Materials Science \& Engineering and in the Department of Electrical \& Computer Engineering at the Michigan Technological University where he runs the Open Sustainability Technology Research Group. His research concentrates on the use of open source appropriate technology to find collaborative solutions to problems in sustainability and poverty reduction. His research spans areas of electronic device physics and materials engineering of solar photovoltaic cells, and RepRap 3-D printing, but also includes applied sustainability and energy policy. His research is regularly covered by the international and national press and it is continually ranked in the top $0.1 \%$ on Academia.edu (over 400 publications). He is the editor-in-chief of HardwareX, a journal dedicated to open source scientific hardware. 


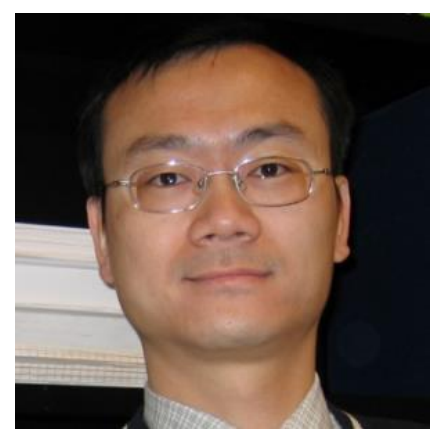

John Zhai is a Professor in the Department of Civil, Environmental and Architectural Engineering (CEAE) at University of Colorado at Boulder. He has a unique and integrated background in both Mechanical and Architectural Engineering with an Engineering Doctor degree in Fluid Mechanics (Tsinghua University, 1999) and a Ph.D. in Building Technology (MIT, 2003). He has been actively engaged in research activities in the field of fluid/thermal science and building/energy/environment technology since 1994. As a primary researcher, he has completed a great number of notable research and consulting projects on diverse building energy and environment topics and published over 130 technical papers in reputed journals and conferences. He is also Graduate Advisor at Purdue University, Adjunct Professor at School of Environmental Science and Technology at Tianjing University, China, The "Sea-Sky" University Professor at School of Civil Engineering at Dalian University of Technology, China. He also held a "Senior Fellow" position at the Rocky Mountain Institute (a renowned sustainable building design and consulting firm). He is Associate Editor of Energy and Building Journal and The Open Civil Engineering Journal, Guest Editor for Building and Environment Journal, World Review of Science, Technology and Sustainable Development Journal, Guest Editor and Editorial Board Member for Building Simulation: An International Journal, and Editorial Board Member for Journal of Building Physics, Indoor and Built Environment Journal, Journal of Energy, and Journal AIMS Energy. 